\title{
Interfacial Tension of a Decomposed Biopolymer Mixture
}

\author{
E. Scholten, ${ }^{\dagger, \neq}$ R. Tuinier,,,,$+ \S$ R. H. Tromp, ${ }^{\S}$ and H. N. W. Lekkerkerker ${ }^{\dagger}$ \\ Van 't H off Laboratory, Debye Research Institute, University of Utrecht, \\ Padualaan 8, $3584 \mathrm{CH}$ Utrecht, The Netherlands, and NIZO food research, \\ P.O. Box 20, 6710 BA Ede, The Netherlands
}

Received September 14, 2001. In Final Form: November 26, 2001

\begin{abstract}
Many biological systems as well as food products contain both proteins and polysaccharides, which are often thermodynamically incompatible. In this communication, measurements are presented of theinterfacial tension in a segregated polysaccharide/protein mixture. The interfacial tension is an important quantity in determining the phase separation kinetics and the response to shear. As the protein/polysaccharide system, an aqueous gelatin/dextran mixture in 1.0 M sodium iodide was chosen to suppress gelation and achi eve a low density difference between the coexisting phases. First, the phasethreshold was determined by making dilution lines. From the relative volumes of the coexisting phases, the location of the critical point was estimated. Interfacial tensions of phase-separated mixtures, varying in distancefrom thecritical point, were measured using the spinning drop method. The interfacial tension cl ose to the critical point was less than $1 \mu \mathrm{N} / \mathrm{m}$, and it increased, in a nonlinear fashion, to a value of up to $20 \mu \mathrm{N} / \mathrm{m}$ farther from the critical point. For the scaling relation of the interfacial tension with the density difference and the polymer concentration difference, we found scaling exponents of $2.7 \pm 0.3$ and $1.5 \pm 0.1$, respectively, which are in agreement with the critical mean-field scaling exponents of 3 and 3/2, respectively.
\end{abstract}

\section{Introduction}

In biological and food systems, polysaccharides and proteins are often jointly present. When a certain concentration of these biopolymers in themixture is exceeded, phaseseparation often results. ${ }^{1}$ When thepolysaccharides are charged and the $\mathrm{pH}$ is such that the two biopolymers are oppositely charged, complexation can be responsible for decomposition. Alternatively, segregation of effectively repulsive biopolymers (equally charged or uncharged) can take place. In the latter situation, each phase becomes enriched in either polysaccharide or protein. During the past decade, a significant amount of work has been performed on studying the stability of polysaccharide/ protein mixtures, as well as developing quantitative descriptions of the phase boundaries; seer f 2 for a recent review. These studies contribute to an understanding of the concentrations at which, and the conditions under which, phase separation processes are expected to occur. If phase separation takes place, the driving force for decomposition must overcometheaccompanying increase in interfacial free energy, which equals the product of the interfacial tension and thetotal interfacial area associated with the phase separation. Therefore, the interfacial tension between the coexisting phases of the decomposed polysaccharide/protein system is of fundamental interest. The order of magnitude of the interfacial tension can be estimated from thescaling relation $\gamma=\mathrm{O}\left(\mathrm{kT} / \xi^{2}\right),{ }^{3-5}$ where $\gamma$ is theinterfacial tension and $\xi$ is the width of the region

* Corresponding author. Present affiliation: Forschungszentrum J ülich, Institut für F estkörperforschung, Weiche Materie, 52425 J ülich, Germany. Electronic mail: r.tuinier@fz-juelich.de.

† Debye Research Institute.

₹ Present affiliation: Food Physics, Department of Food Technol ogy and Nutritional Sciences, Wageningen University, Bomenweg 2, 6703 HD Wageningen, The Netherlands.

$\S \mathrm{NIZO}$ food research. 145.

(1) Grinberg, V. Y.; Tolstoguzov, V. B. Food Hydrocol loids 1997, 11,

(2) Doublier, J .-L.; Garnier, C.; Renard, C.; Sanchez, C. Curr. Opin. Colloid Interface Sci. 2000, 5, 184.

(3) de Gennes, P. G. Scaling Concepts in Polymer Physics; Cornell University Press: Ithaca, NY, 1979. in which the concentration of the components differ from their bulk values in the coexisting phases. Theinterfacial width is supposed to have a value of a few molecular diameters. Biopolymers usually have sizes in the range of 5-100 nm, corresponding to a range of interfacial tension of $1-100 \mu \mathrm{N} / \mathrm{m}$. These values areextremel y small in comparison with values for the interfacial tension between gases and liquids of atoms or low-molecular-mass substances, which liebetween 1 and $100 \mathrm{mN} / \mathrm{m}$. I n addition to being a quantity that is of fundamental interest, the interfacial tension is al so one of the key parameters that influences the phase separation rate. ${ }^{6-9}$ Irrespective of whether onefocuses on the spinodal decomposition, ${ }^{6,7}$ the stability of a film between two droplets, ${ }^{8}$ or the breakup of a liquid cylinder, ${ }^{9}$ the interfacial tension al ways plays a central role.

Several studies ${ }^{10-15}$ have been directed at analyzing and interpreting the interfacial tension in demixed polymer solutions. Themagnitudeof theinterfacial tension depends on how far the system deviates from the critical point, near which values as small as $1 \mu \mathrm{N} / \mathrm{m}$ have been reported. ${ }^{10,11}$ Critical exponents were determined and found to be quite reproducible for homopolymers, which led Heinrich and Wolf to propose a master curve for polystyrene. ${ }^{11}$ Recently, however, for random copolymers,

(4) Rowlinson, J . S.; Widom, B. Molecular Theory of Capillarity; Clarendon Press: Oxford, U.K., 1984

(5) Vrij, A. Physica A 1997, 235, 120

(6) Siggia, E. D. Phys. Rev. A 1979, 20, 595

(7) Verhaegh, N. A. M.; Lekkerkerker, H. N. W. Phase transitions in colloidal suspensions. In Proceedings of the International School of Physics 'Enrico Fermi'; I OS Press: Amsterdam, 1997.

(8) I vanov, I. B.; Kralchevsky, P. A. Coll loids Surf. A 1997, 128, 155

(9) deH oog, E. H. A.; Lekkerkerker, H. N. W.J . Phys. Chem. B 2001 105 (47), 11636 Lekkerkerker, H. N. W.; de Hoog, E. H. A. Physica A 2001, 298, 69.

(10) Shinozaki, K.; van Tran, T.; Saito, Y.; Nose, T. Polymer 1982 23, 728.

(11) Heinrich, M.; Wolf, B. A. Polymer 1992, 33, 1926.

(12) Heinrich, M.; Wolf, B. A. Macromolecules 1992, 25, 3817.

(13) Enders, S.; Huber, A.; Wolf, B. A. Polymer 1994, 35, 5743.

(14) Enders, S.; Wolf, B. A.; Binder, K. J . Chem. Phys. 1995, 103, 3809.

(15) Schneider, A.; Wolf, B. A. Polymer 2000, 41, 4089 
significant differences in thecritical exponents of various systems have been found. ${ }^{15}$

The interfacial tension of decomposed polysaccharide/ protein mixtures is, in contrast to the phase stability of these systems, an aspect to which limited attention has yet been paid. Tolstoguzov et al . ${ }^{16}$ measured the interfacial tension of gelatin/dextran aqueous mixtures using a capillary rise method and reported a value of $27 \mu \mathrm{N} / \mathrm{m}$. Wolf et al. ${ }^{17}$ applied shear to a phase-separated aqueous gellan/ $\kappa$-carrageenan mixture, and from the shape of the droplets, they estimated an interfacial tension of $7.5 \pm$ $1.4 \mu \mathrm{N} / \mathrm{m}$. Using the samemethod, Stokes et al. ${ }^{18}$ measured theinterfacial tension of theinterfacein a phaseseparated gelatin/maltodextrin system, resulting in values in the range of $50-100 \mu \mathrm{N} / \mathrm{m}$. These reported values correspond to the expected order of magnitude.

Here, we present measurements of the interfacial tension of the interface between the phases in a decomposed gelatin/dextran mixture in $1.0 \mathrm{M}$ sodium iodide. Recent investigations suggest ${ }^{19}$ that the phase separation in gelatin/dextran mixtures is mainly entropy-driven. The protein gelatin is frequently used in low fat products or as a binder in yogurt, and it is a material that makes it possible to encapsulate (pharmaceutical) ingredients in capsules having a neutral taste. Gelatin is obtained from thefibrous protein collagen, extracted mai nly from bones or hides of various animals, using chemical/thermal processing. It is able to undergo a conformational coil/ helix transition, where chains in the helix conformation can form reversible gels. ${ }^{20}$ Dextran is a polysaccharide produced by the bacterium Leuconostoc mesenteroides, which, after its discovery in the 19th century, became of significance as a thickener in the food industry. Most dextrans mainly consist of $\alpha(1 \rightarrow 6)$ linked glucoses and a small fraction of the glucoses linked via $\alpha(1 \rightarrow 3)$ bonds. Both gelatin and dextran are approved food ingredients.

Sodium iodide was used as a salt to suppress gelatin gelation as well as to achieve a small density difference between the coexisting phases; iodidehas a higher affinity to bind to gelatin than to dextran, thereby making the (upper) gelatin-rich phase heavier. Weused thespinning drop method for measuring the interfacial tensions. It has been demonstrated that this method is well-suited for measuring very low interfacial tensions. ${ }^{21}$ Thespinning drop method has been used successfully in recent years to determine the interfacial tensions in demixed colloid/ polymer mixtures, ${ }^{22-24}$ yielding tensions with values of a few micronewtons per meter.

\section{Experimental Section}

Materials. A purified low-molar-mass fraction of gelatin was a gift from DFG-Stoess, Germany, and dextran was purchased from Fluka. The molar mass (distribution) of the samples was determined using a combination of size-exclusion chromatography (SEC) and multi-angle laser light scattering (MALLS) at

(16) Tolstoguzov, V. B.; Mzhel'sky, A. I .; Gulov, V. Ya. Colloid Polym. Sci. 1974, 252, 124.

(17) Wolf, B.; Scirocco, R.; Frith, W.J .; Norton, I. T. Food Hydrocoll loids 2000, 14, 217.

(18) Stokes, J . R.; Wolf, B.; Frith, W. J . J . Rheol. 2001, 45, 173.

(19) Edelman, M. W.; van der Linden, E.; de Hoog, E.; Tromp, R. H. Biomacromolecules 2001, 2, 1148.

(20) Normand, V.; Muller, S.; Ravey, J .-C.; Parker, A. Macromol ecules 2000, 33,1063

(21) Kegel, W. K.; van Aken, G. A.; Bouts, M. N.; Lekkerkerker, H. N. W.; Overbeek, J. Th. G.; de Bruin, P. L. Langmuir 1993, 9, 252.

(22) Vliegenthart, G. A.; Lekkerkerker, H. N. W. Prog. Colloid Polym.

Sci. 1997, 105, 27

(23) de Hoog, E. H. A.; Lekkerkerker, H. N. W. J . Phys. Chem. B 1999, 103, 5274 . 2369.
NIZO F ood Research, as described in refs 25 and 26 . The gelatin had a number-averagemol ar mass, $M_{n}$, of $24 \mathrm{~kg} / \mathrm{mol}$ and a weightaveraged molar mass, $M_{w}$, of $41 \mathrm{~kg} / \mathrm{mol}$, resulting in a polydispersity, expressed as $M_{w} / M_{n}$, of 1.7. The investigated dextran sample had a number-average radius of gyration $\left(R_{g}\right)$ in aqueous solution of $20 \pm 1 \mathrm{~nm}$, a $M_{n}$ of $260 \mathrm{~kg} / \mathrm{mol}$, and a $M_{w}$ of $387 \mathrm{~kg} / \mathrm{mol}$ $\left(\mathrm{M}_{\mathrm{w}} / \mathrm{M}_{\mathrm{n}}=1.49\right)$.

Methods. Preparation of thePolysacchari ded Protein Mixtures. Both gel atin and dextran were simultaneously dissolved in 1.0 $\mathrm{M}$ sodium iodide ( $\mathrm{Nal}$ ). Sodium azide $(0.03 \%)$ was added as an antimicrobial agent. The mixtures, which had a pH of 6.0, were kept at room temperaturefor at least $1 \mathrm{~h}$, after which thesamples were heated to $60^{\circ} \mathrm{C}$ and kept at that temperature for $30 \mathrm{~min}$. They were then vigorously shaken to obtain a homogeneous mixture. All subsequent experiments were made at room temperature.

Spinning Drop Method. To measure the interfacial tension of the gelatin/dextran mixtures, a spinning drop tensiometer was used, ${ }^{22,23}$ which included a spinning drop tube with a diameter of $4 \mathrm{~mm}$ and a length of $4 \mathrm{~cm}$ (see ref 23 for details). The highdensity (dextran-rich) phase, with a volume of $\sim 1 \mathrm{~mL}$, was injected into the spinning drop tube, which was closed on one side with a Teflon stopper, using a glass capillary. The volume of the low-density droplets, injected with a microsyringe, was 1-2 $\mu \mathrm{L}$. Subsequently, thetubewas inserted intothetensiometer and rotated about its axis. Thus, a centrifugal field was applied to deform the low-density droplet in the high-density matrix. The applied spinning frequency was high enough that the drop was spinning sufficiently fast about its horizontal axis to neglect acceleration dueto gravity. In thecentrifugal field, the drop was el ongated al ong the rotational axis until a certain equilibrium deformation was achieved where the Laplace pressure over the interfacewas balanced by the centrifugal pressure. At sufficiently high rotational speeds, the length of the droplet will exceed four times the dropl et diameter, and it follows ${ }^{27}$ that the droplet will closel y resemble a cylinder with spherical caps. In that case, the Vonnegut equation will be valid 28

$$
\gamma=\frac{\Delta \rho \omega^{2} R^{3}}{4}
$$

where $\omega$ is thespinning frequency, R is the radius describing the curvature on the outside of the el ongated droplet, and $\Delta \rho$ is the density difference between the coexisting phases. It was al ways verified during the measurements that the droplet had a length that exceeded 8R. To determine the interfacial tension, at least threedroplets werespun several times at five or six frequencies. The rotational speeds were measured with an optical sensor, and the observed dimensions (length and diameter) of the el ongated droplets weremeasured with a micrometer. As a result of the curvature of the tube, the dimensions of the droplet are slightly different from its actual size. ${ }^{29}$ Theactual droplet radius, $R_{d}$, was calculated from the apparent drop radius, $R_{d a}$, as $R_{d}=$ $R_{d a} n_{a} / n_{1}$, where $n_{1}$ and $n_{a}$ are the refractive indices of the liquid surrounding the drop and of the phase outside the tube, respectively. The refractive index of the high-density phase was determined using a refractometer. After each measurement, the rotational speed was increased, and the droplet was allowed to deform for about $1 \mathrm{~h}$, after which the shape of the droplet no longer changed. Compared to previous reports, ${ }^{22,23}$ the equilibration time required here was very small. This allowed the measurements at different rotational speeds to be carried out within a relatively short period of time.

Construction of the Phase Diagram. Samples were prepared that segregated and diluted with solvent (1.0 M Nal). In this way, the gelatin/dextran rati o was held constant. After dilution, the solution was mixed and shaken, and it was then investigated whether thesamples still phase-separated. Bel ow a certain total

(25) Hoffmann, M. A. M.; Sala, G.; Olieman, C.; de Kruif, C. G. J . Agric. Food Chem. 1997, 45, 2949.

(26) Tuinier, R.; Ten Grotenhuis, E.; de Kruif, C. G. Food Hydrocolloids 2000,14, 1.

(27) Vonnegut, B. Rev. Sci. Instrum. 1930, 13, 6.

(28) Princen, H. M.; Zia, I.Y. Z.; Mason, S. G. J . Colloid InterfaceSci. 1967, 23, 99 .

(29) Coucoulas, L. M. J . Colloid Interface Sci. 1983, 93, 281. 


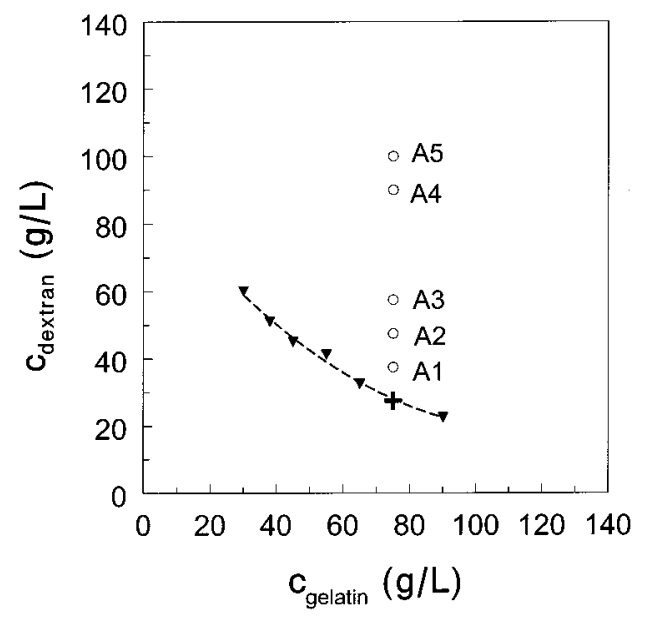

Figure 1. Phase diagram of mixture of gelatin and dextran in $1.0 \mathrm{M} \mathrm{Nal}$. Solid triangles indicate the estimated phase threshold values along a dilution line. The estimated critical point is identified by the cross $(+)$. The open circles refer to mixtures for which, after phase separation, the interfacial tension has been measured.

Table 1. Interfacial Tension, Concentration, and Density Difference for the Gelatin/Dextran Phase-Separated Mixtures Presented as Open Circles in Figure 1

\begin{tabular}{ccccc}
\hline $\begin{array}{c}\text { sample } \\
\text { number }\end{array}$ & $\begin{array}{c}\text { gelatin } \\
\text { concentration } \\
(\mathrm{g} / \mathrm{L})\end{array}$ & $\begin{array}{c}\text { dextran } \\
\text { concentration } \\
(\mathrm{g} / \mathrm{L})\end{array}$ & $\begin{array}{c}\Delta \rho \\
(\mathrm{g} / \mathrm{L})\end{array}$ & $\begin{array}{c}\gamma \\
(\mu \mathrm{N} / \mathrm{m})\end{array}$ \\
\hline A1 & 75.1 & 37.6 & $1.1 \pm 0.1$ & $0.5 \pm 0.1$ \\
A2 & 74.8 & 47.5 & $1.8 \pm 0.1$ & $2.4 \pm 0.3$ \\
A3 & 75.2 & 57.6 & $2.7 \pm 0.1$ & $5.9 \pm 0.5$ \\
A4 & 75.2 & 90.0 & $4.0 \pm 0.1$ & $15.7 \pm 0.7$ \\
A5 & 75.0 & 100.0 & $4.1 \pm 0.1$ & $19.3 \pm 0.8$
\end{tabular}

biopolymer concentration, themixtures remained homogeneous. The threshold concentrations were then taken to be situated halfway between the lowest separating and highest nonseparating compositions. In the dilution series, steps of $5-10 \mathrm{~g} / \mathrm{L}$ were made.

Density Measurements. The densities of the both phases of the decomposed system were determined with an Anton Paar DMA 5000 density meter. The difference in density between the two phases is required for the interfacial tension to be calculated, as well as for the (critical) scaling behavior to be studied. The densities of the samples were measured at least four times to also determine the statistical error.

\section{Results and Discussion}

Phase Behavior of Gelatin/Dextran Mixtures. Aqueous systems of gelatin/dextran in $1.0 \mathrm{M} \mathrm{Nal}$ at sufficient concentrations show phase separation into two clear fluid phases, separated by a sharp interface. ${ }^{19,30}$ The upper phase is (relatively) concentrated in gelatin, and the lower phase is enriched in dextran. The interface can be described as appearing sharp to the eye. The phasediagram was constructed along the dilution lines by determining the concentrations above which phase transition takes place. For gelatin/dextran mixtures in $1.0 \mathrm{M}$ $\mathrm{Nal}$, the resulting phase diagram is plotted in Figure 1. In this figure, the solid triangles refer to those points on the di lution line where the stability threshold is crossed. The cross (+) refers to the estimated critical point, determined from the position at the threshold where the volumes of the coexisting phases are equal. An estimation of the overlap concentration, $\mathrm{c}^{*}$, of dextran can be made using the weight-average molar mass of $387 \mathrm{~kg} / \mathrm{mol}$ and

(30) Tromp, R. H.; Rennie, A. R.; J ones, R. A. L. Macromolecules 1995, 28, 4129.

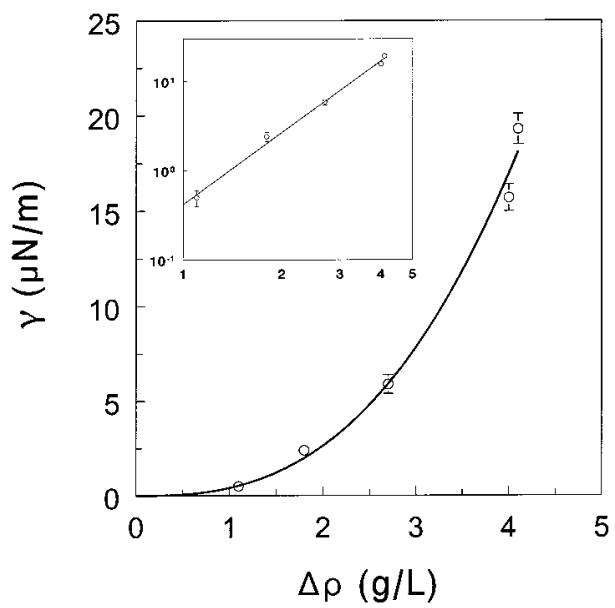

Figure 2. Interfacial tension of the systems referred to in Figure 1 as a function of the density difference. The drawn curve is the best fit to the scaling relation $\gamma=q(\Delta \rho)^{p}$ (see text). The scaling relation is plotted logarithmically in the inset.

the average radius of gyration of $20 \mathrm{~nm}$ to give $\mathrm{c}^{*} \approx 19$ $\mathrm{g} / \mathrm{L}$. The dextran concentration at thephaselinein $\mathrm{Figure}$ 1 lies above this val ue for the sampl es investigated which indicates that phase separation takes place in the semidilute range of dextran concentrations. The phase diagram was constructed to determine the critical point, which is required to study the density difference and concentration dependence of the interfacial tension.

Interfacial Tension Data. To determine the interfacial tension, samples were allowed to phase separate, and the coexisting phases were then put into the spinning drop tensiometer, as described in section 2. An overview of the results of the measurements made, including thestatistical error in themeasured quantities, is given in Table1. Theinterfacial tensions werecal culated with eq 1.

Because $\Delta \rho \equiv 0$ at the critical point, the value for the density difference, $\Delta \rho$, is indicative of the quench depth, i.e., how far the system is from the critical point. For the gelatin/dextran system, thegel atin concentration was kept constant, and only the dextran concentration was varied to simplify the analysis. Because the density differences weresmall (on theorder of grams per liter), therotational speeds had to be very high (at least $250 \mathrm{rad} / \mathrm{s}$ ) to deform the droplets such that they were sufficiently el ongated. As can be seen in Table 1, the interfacial tension close to the critical point is very small, namely, $0.5 \pm 0.1 \mu \mathrm{N} / \mathrm{m}$ for system A1 in Table 1 . This value increases to values of $\sim 20 \mu \mathrm{N} / \mathrm{m}$ farther from the critical point. These values are smaller than those reported by Stokes et al. ${ }^{18}$ and can be explained by the fact that the maltodextrin they used had a relatively low molar mass. Theoretically, an estimate of theinterfacial tension can be obtained from the scaling relation $\gamma \sim \mathrm{kT} / \xi^{2} \cdot{ }^{3-5} \mathrm{~F}$ or thesize of themolecules involved here, wecan takethedextran radius of gyration toestimate the order of magnitude of the interfacial tension. For $R_{g}$ $=20 \mathrm{~nm}$ (the average value for dextran), we find at 298 $\mathrm{K}$ an estimate for the interfacial tension of $\sim 10 \mu \mathrm{N} / \mathrm{m}$, which corresponds to the magnitude of the values found in the gelatin/dextran systems investigated, as reported in Table 1.

Interfacial Tension, Scaling Behavior. The set of data we obtained allows us to investigate the scaling behavior of thegelatin/dextran system. Theeffect of deeper quenching was studied by increasing the overall dextran concentration while keeping the overall gelatin concentration constant. In Figure2, weplot theinterfacial tension 


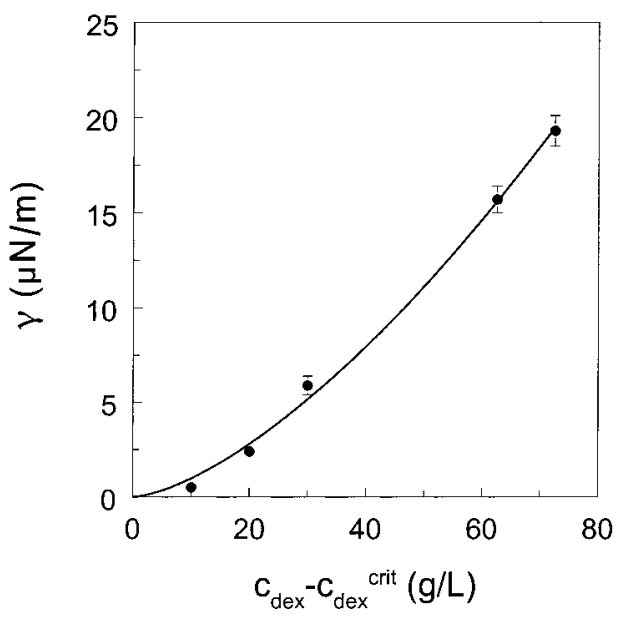

Figure 3. Interfacial tension of the systems referred to in Figure 1 as a function of the difference of the dextran concentration from that at the critical point. The full curve is a best fit of the scaling relation $\gamma=\mathrm{a}\left(\mathrm{c}_{\mathrm{dex}}-\mathrm{c}_{\mathrm{dex}}^{\mathrm{crit}}\right)^{\mathrm{b}}$ (see text).

as a function of the density difference, $\Delta \rho$, between the coexisting phases. The dependence of $\gamma$ on $\Delta \rho$ is nonlinear. The data were fitted to the relation $\gamma=\mathrm{a}(\Delta \rho)^{\mathrm{b}}$, for which $a=0.4 \pm 0.2$ and $b=2.7 \pm 0.3$ were found as best-fit parameters (see the fit in Figure 2). Thescaling exponent of $2.7 \pm 0.3$ is cl ose tothat given by themean-field relation ${ }^{4}$

$$
\gamma \sim(\Delta \rho)^{3}
$$

The Ising model predicts a value of $3.88,{ }^{4}$ which is significantly larger than thevaluewefound. For a demixed polystyrene solution, Shinozaki et al. ${ }^{10}$ and Heinrich and Wol f11 reported val ues of 3.3-3.9 for thecritical exponent, which agrees well with the Ising model. Schneider and Wolf, however, recently found ${ }^{15}$ for random copolymers that the critical exponent can vary from 2.5 to 4.6. These latter findings were explained in terms of a more suitable way of arranging the copolymer monomeric units in the interface. Because gelatin is a copolymer as well, deviations from the Ising critical exponent are to be expected.

For the case of depletion-induced phase separation of a colloid/polymer mixture, Brader and E vans ${ }^{31}$ performed density functional theory calculations of the interface. In their osmotic equilibrium theory, theinvestigated system was hel $d$ in equilibrium with a hypothetical reservoir that contained only polymer. Their results for the interfacial tension correspond to eq 2 .

They also investigated the scaling of the interfacial tension with the polymer concentration in the reservoir and found the relationship

$$
\gamma \sim\left(c_{p}^{R}-c_{p}^{R, c r i t}\right)^{3 / 2}
$$

where $c_{p}^{R}\left(c_{p}^{R, c r i t}\right)$ is the (critical) polymer concentration in the reservoir. In our experimental system, it is hard to determine the effective free volume for the polymers. Therefore, we can only compare these scaling results for the polymer reservoir concentration $\left(c_{\mathrm{p}}^{R}-c_{\mathrm{p}}^{R \text {, crit }}\right)$ with $\left(c_{\text {dex }}\right.$ $\left.-C_{\text {dex }}^{\text {crit }}\right)$, where $c_{\text {dex }}^{\text {crit }}$ is the concentration of dextran in the critical point, for the data points in the phase diagram of Figure 1. Results for the interfacial tension as a function of $\left(c_{d e x}-c_{d e x}^{\text {crit }}\right)$ are plotted in Figure 3. Obviously, the dependenceis again rather nonlinear. Thecurvewas fitted to the scaling relation $\gamma=\mathrm{q}\left(\mathrm{c}_{\mathrm{dex}}-\mathrm{c}_{\mathrm{dex}}^{\text {crit }}\right)$, for which the best-fit values are $q=0.033 \pm 0.002$ and $p=1.5 \pm 0.1$

(31) Brader, J . M.; Evans, R. Europhys. Lett. 2000, 49, 678.

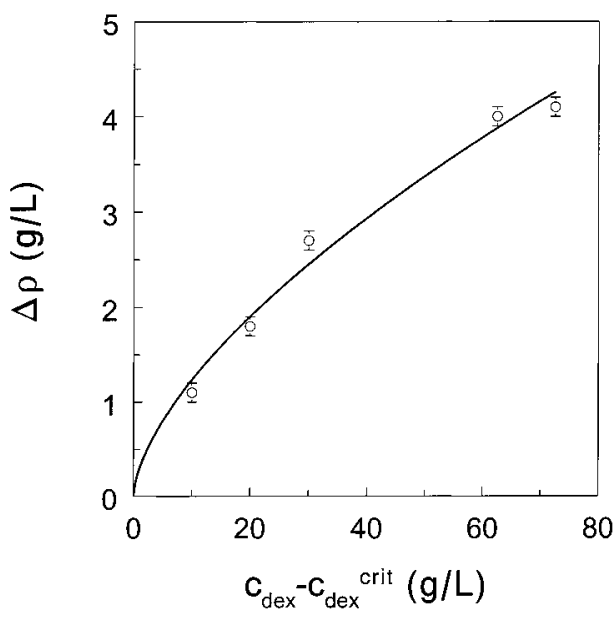

Figure 4. Density difference as a function of the difference of the dextran concentration from that at the critical point.

(the fit is plotted as the full curve). The exponent of 1.5 matches the theoretical result of $3 / 2$.

For the interface of a critical mixture, there is also a scaling relation for the concentration difference with the critical point concentration (denoted as $\Delta \mathrm{c}$ ) and the density difference, $\Delta \rho$, namely, $\Delta \rho \sim(\Delta \mathrm{c})^{\mathrm{z}}$, with $\mathrm{z}=0.5$ in a meanfield approach (combining eqs 2 and 3 ) and $z=0.32$ for the Ising model. In Figure 4, we pl ot $\Delta \rho$ as a function of $c_{\text {dex }}-c_{\text {der }}^{\text {crit }}$ which we take as $\Delta c$. From the experimental data, it follows that $z=0.55 \pm 0.10$, which is in agreement with the mean-field value but above the Ising model prediction. For all determined scaling exponents, wethus find that the experimental results agree with mean-field critical scaling predictions. Odijk ${ }^{32}$ argued that, for a mixture of small proteins and large polymers, a meanfield description suffices. This might explain why the results presented for the interface can be described by a mean-field theory as well.

Care should be taken, however, when comparing the measured scaling exponent data with theoretical values. First, there is some uncertainty in the location of the critical point; it is not certain whether there is a welldefined critical point for a decomposed mixture of two polydisperse biopolymers. The critical point will be a projection of a critical line in the phase diagram with a high dimensionality onto a two-dimensional plot. Further, the determination of the critical point was made using an operational definition: thelocation at thethreshold where the volumes of the coexisting phases are equal. Another issue that might explain discrepancies is that the quench depth at which the exponent is determined might differ from the region that is sufficiently close to the critical point to expect critical (scaling) behavior.

The dependence of theinterfacial tension on the density difference differs from the results obtained using a model colloid/polymer mixture by De Hoog and Lekkerkerker. ${ }^{23}$ They found an approximately linear dependence of $\gamma$ on $\Delta \rho$, which can be explained by the fact that the relative density difference was very large in their system, indicating that their system is relatively farther from thecritical point. From the results of Chen et al., ${ }^{24}$ it follows that the exponent is close to 3.5 , which is between the mean-field (3) and I sing model predictions (3.88). An important issue here is determining which system is closest to the critical point. To quantify the distance from the critical point, the density difference $\Delta \rho$ can benormalized by the density at

(32) Odijk, T. Macromolecules 1996, 29, 1842; Physica A 2000, 278, 
the critical point, $\rho^{\text {crit }}$. The densities of dextran, gelatin, and the $1.0 \mathrm{M} \mathrm{Nal}$ solvent could be estimated from the density measurements as 1605, 1367, and $1110 \mathrm{~g} / \mathrm{L}$, respectively, giving an estimation for the density at the critical point of $\sim 1150 \mathrm{~g} / \mathrm{L}$. This means that $\Delta \rho / \rho^{\text {crit }}$ is al ways smaller than 0.005 , whereas the values for $\Delta \rho / \rho^{\text {crit }}$ in the systems of both De Hoog and Lekkerkerker ${ }^{23}$ and Chen et al. ${ }^{24}$ were in the range from about 0.1 to 0.4 . The measurements on the decomposed gel atin/dextran mixture reported hereare, therefore, relativel y closer tothecritical point. The determination of the scaling exponents allows the scaling relations to be used to describe, for instance, the phase separation kinetics and response to shear of (phase-separating) gelatin/dextran mixtures.

\section{Conclusions}

Using thespinning drop method, theinterfacial tension of phase separated gelatin/dextran mixtures was determined. The interfacial tension close to the critical point was determined to be close to $1 \mu \mathrm{N} / \mathrm{m}$, and it increased to values of $20 \mu \mathrm{N} / \mathrm{m}$ farther from the critical point. From thesemeasurements, wecan concludethat themagnitudes of theinterfacial tension can becompared with thosefound in phase-separated model colloid/polymer and polymer mixtures and that thesevalues are low compared to those for an atomic gas/liquid interface $(1-100 \mathrm{mN} / \mathrm{m})$. The scaling behavior of the interfacial tension close to the critical point was compared with theoretical predictions. Experimental scaling exponents were in reasonable agreement with critical mean-field values.

Acknowledgment. This work was sponsored by the NWO-Unilever program. Special purified gelatin fractions were kindly supplied by DF G-Stoess. We are indebted to M. W. Edelman, E. H. A. de Hoog, and A. Vrij for useful discussions and to S. R. Williams and E. ten Grotenhuis for a critical reading of the manuscript. E. Sallomons, C. Pathmamanoharan, and J. Suurmond are thanked for technical assistance. R.T. thanks H. J . Klok and C. G. de Kruif for helpful discussions on the gelatin/dextran system.

LA0114373 